\title{
INTERVENCIÓN CONDUCTUAL EN UN CASO DE RETRASO MENTAL
}

\section{Carmen Vives Montero}

Universidad de Granada

\section{RESUMEN}

En este estudio se describe el tratamiento aplicado a nifia con 5 años con retraso mental, que a pesar de haber recibido tratamiento anterior carecia de repertorio verbal, y presentaba deficits conductuales en la mayorfa de la áreas evaluadas, asi como problemas de conducta. Después de la evaluación se inicló el tratamiento siguiendo los procedimientos del Análisis de Conducta Aplicado dirigidos a incrementar los repertorios de conductas prerreguisitas, reforzamiento de conductas Incompatibles con las conductas problema y posteriomente, se Inicio el moldeamiento para enseñarle a imitar palabras y grupos consonánticos. Esto le permitió progresar en la adquisición de tactos, mandos $\theta$ intraverbales. También se plantearon algunos objetivos de las áreas cognitiva, motora y social en la que se requiní la participación de los padres. Después de 14 meses de tratamlento se comprobó una

Correspondencla: M Carmen Vives Montero. Departamento de Personalidad, Evaluación y Tratamiento Psicológico. Facultad de Psicología. Campus Universitario de La Cartuja. 18071 Granada. Teléfono: 958-243751. e-mall: cvives Ougr.es

Agradecimientos: Deseo expresar ml agradecimiento a Karen Shashok por la traducción del resumen al inglés y a Antonio Femández Parra y Rafael Ferro por sus comentarios. 
ampliación en el repertorio verbal de la niña y se registró un incremento en todas áreas evaluadas con la Guía Portage. Aquí se demuestra como al incluir la adquisición de las conductas prerrequisitas para el aprendizaje entre los objetivos de tratamiento y la aplicación de los procedimientos de intervención conductuales permitieron que una niña con retraso consiguiera un repertorio verbal básico que no había alcanzado mediante el tratamiento tradicional más prolongado que habia recibido anteriormente.

Palabras clave: RETRASO MENTAL, TRATAMIENTO, ANÁLISIS CONDUCTUAL APLICADO

\section{SUMMARY}

We report the treatment used for a 5-year-old girl with developmental delay who, despite previous treatment, had no verbal repertoire and displayed behavioral deficits in most areas evaluated as well as behavioral problems. After the evaluation, treatment was started with applied behavioral analysis procedures aimed at increasing repertoires of prerequisite behaviors and reinforcing behaviors incompatible with problem behaviors. Subsequently, shaping was begun to teach the girl to imitate words and consonant groups. This made progress possible in the acquisition of tacts, mands and intraverbals. Objectives were also chosen in the areas of cognitive, motor and social functioning which required particlpation by the parents. After 14 months of treatment the girl's verbal repertoire had expanded, and gains were see in all areas evaluated with the Portage Guide. We show here that including the acquisition of prerequisite behaviors among the treatment aims and applying behavioral intervention procedures made it possible for a git with developmental delay to develop a basic verbal repertoire that she had not attained with a longer traditional treatment received proviously.

Key words: MENTAL RETARDANON, TREATMENT, APPLIED BEHAVIORAL ANALYSIS 
La manera de abordar el estudio y definición del retraso mental o retardo en el desarrollo ha sido diversa. Se pueden diferenciar cuatro posiciones conceptuales: 1 . La definición basada en el cociente de inteligencia (Cl). 2. La definición basada en la conducta adaptativa (CA). 3. La definición de la American Association of Mental Retardation (AAMR, 2002) que recoge los dos índices anteriores. 4. La definición del Análisis de Conducta Aplicado basada en déficits y excesos específicos (Bijou, 1976).

Algunos criterios de definición propuestos por la American Association of Mental Retardation han sido incorporados en el DSMIV y CIE-10. Tal y como se recoge en el DSM-IV-TR (APA, 2002), el Retraso Mental se caracteriza por un funcionamiento intelectual significativamente por debajo de la media, que coexiste con limitaciones en dos o más de las siguientes áreas de habilidades adaptativas: comunicación, cuidado personal, vida doméstica, habilidades sociales, utilización de los servicios de la comunidad, autodirección, salud y seguridad, rendimiento académico funcional, ocio y trabajo. Se manifiesta antes de los 18 años.

Tradicionalmente el diagnóstico del retraso en el desarrollo o retraso mental se ha basado en la medición de la capacidad intelectual general $(\mathrm{Cl})$, obtenido mediante los tests de inteligencia normalizados. Ante las críticas que recibió el diagnóstico tradicional (Gil, 1988; Luciano, López y Vives, 1997), el Análisis de Conducta Aplicado propuso la alternativa conocida como la Evaluación Conductual. Esta aproximación al retraso se centra en la evaluación de los excesos y los déficits conductuales empleando estrategias de evaluación con referencia a la noma y basada en el criterio de ejecución individual (Bijou, 1976). Otra de sus aportaciones ha sido la forma de evaluar la conducta verbal mediante la observación y la recogida de información sobre el repertorio del niño en las distintas operantes verbales (Sundberg y Michael, 2001), dado que la información aportada por los tests tradicionales no proporciona todos los datos necesarios para la planificación de la intervención.

La mayoría de los niños con retraso en el desarrollo presentan déficits en el repertorio verbal, que pueden variar desde la ausencia total de repertorios verbales hasta dificultades leves de vocabulario o de articulación. El comportamiento verbal se con- 
sidera una conducta necesaria para el desarrollo de otras competencias y por esta razón uno de los objetivos principales en el tratamiento de los niños con retraso radica en incrementar el lenguaje funcional.

A nivel práctico se hace una distinción entre la conducta verbal productiva y la receptiva. La conducta verbal receptiva se refiere a la comprensión del lenguaje de otros y la productiva al empleo del lenguaje para interactuar con los demás. Según Luciano (1984) en la conducta verbal productiva se diferencian dos niveles: la conducta ecoica y la funcional. Esta conducta ecoica supone un paso previo al establecimiento de la conducta verbal productiva funcional. En la conducta verbal funcional se incluyen básicamente las relaciones funcionales: Tactos, Mandos, Intraverbales (Skinner, 1957). Estas respuestas verbales pueden ser definidas de la siguiente forma: Respuestas Ecoicas: se refiere a las respuestas de imitación vocal; Tactos: respuestas consistentes en la denominación de objetos 0 acciones; Mandos: hacen alusión a las órdenes o demandas que realiza el hablante; Intraverbales: se refiere a la respuesta verbal a preguntas realizadas por otros hablantes.

Las Técnicas de Modificación de Conducta y la Aproximación Conductual han tenido un gran éxito en la enseñanza de niños con retraso a los que se les había considerado incapaces de aprender (Wicks-Nelson e Israel, 1997) y han demostrado ser más eficaces que otros tratamientos con los que se ha comparado (Green, 1996, y Smith, 1996). Desde el Análisis Conductual Aplicado se han aportado bastantes programas de intervención en lenguaje dirigidos tanto los aspectos formales como los funcionales del lenguaje, como son: Baer, Peterson y Sherman (1967); Drasgow, Halle y Ostrosky (1998); Galindo, Bemal, Hinojosa, Galguera, Taracena, y Padilla (1980); Garcia, Baer y Firestone (1971); Guess, Sailor y Baer (1974, 1976); Harris (1976); Kent, Basil y del Río (1982); Lovaas (1977, 1990); Leaf y McEachin (1998); Maurice, Green y Luce (1996); Sundberg, Endicott, y Eigenheer (2000); Sloane, Johnston y Harris (1968); Watkins, PackTeixteira y Howard (1989); Vives, Luciano y Valero $(2001,2002)$, entre otros.

Con posterioridad al auge de los programas derivados del Análisis de Aplicado aparecieron otros programas para el desarrollo del 
lenguaje denominados interactivos, naturalistas o conversaciones. Sin embargo, en numerosas ocasiones estos programas naturalistas han sido considerados insuficientes para tratar a niños deficientes, por lo cual muchos autores han tenido que incorporar los procedimientos del Análisis de Conducta como el moldeamiento, encadenamiento, desvanecimiento de ayudas, etc. (Del Río, Vilaseca y Gràcia, 1997).

Los tratamientos para la adquisición del lenguaje basados en el Análisis Conductual Aplicado, según Guess, Keogh y Sailor (1986), consideran que la imitación vocal (respuestas ecoicas) es una conducta necesaria para poder intervenir sobre la conducta verbal funcional y por esto el tratamiento parte de la imitación vocal. La intervención se inicia con el moldeamiento de articulaciones nuevas y la diferenciación de respuestas que procede del reforzamiento diferencial del sonido entrenado. Para facilitar el aprendizaje se introducen ayudas y posteriormente se desvanecen. Una vez conseguida la imitación correcta de sonidos y sílabas, mediante encadenamiento se va uniendo sonidos hasta conseguir la imitación de palabras completas. Esto hace posible continuar con el entrenamiento del lenguaje funcional como son: los mandos, los tactos y las intraverbales, etc. (Luciano, 1997, Sundberg y Michael, 2001).

Cuando se producen déficits en la conducta verbal el tratamiento conductual no se centra aisladamente sobre esta área sino que considera también otros aspectos más básicos y amplios del repertorio del niño que son necesarios para que se produzca el aprendizaje de otras conductas. Para iniciar la intervención en el lenguaje el niño debe disponer de un repertorio de conductas prerrequisitas o repertorios básicos para el aprendizaje como son: atención (posición corporal y mantener la mirada), un repertorio imitativo y seguimiento de instrucciones, así como un indice reducido de conductas perturbadoras (Galindo et al.1980; Harris, 1976; Kent, Basil y del Río, 1982; Luciano, 1984, 1997; Luciano y Polaino, 1986; McCoy y Buckhalt, 1981; Ribes, 1972; Vives y Luciano, 1996). Si el niño no ha adquirido estos repertorios básicos con anterioridad, uno de los primeros objetivos del tratamiento se dirigirá a la consecución de dichas conductas prerrequisitas que posteriormente facilitaran el aprendizaje de otros repertorios más complejos. 
En el presente estudio, se expone el tratamiento de una niña con retraso en el desarrollo generalizado y con graves déficits del lenguaje, que anteriormente habla recibido un tratamiento logopédico tradicional durante dos años sin obtener resultados. Para este estudio se apliç un tratamlento conductual, dirigido a objetivos de tratamiento más amplios y fundamentados en los resultados de la Evaluación Conductual realizada previamente.

\section{MÉTODO}

\section{Sujeto}

Elena tenía los 5 años y 10 meses en el momento de iniciar la intervención. Era la segunda hija de una familia de tres hermanos. Sus hermanos hablan seguido un desarrollo normal. La familia pertenecía a una clase social media alta. Los padres acudieron a consulta preocupados principalmente por la ausencia de lenguaje de su hija.

Su madre informó que el embarazo fue normal aunque sufió una depresión durante el tiempo que duró el mismo. Durante el parto la madre tue anestesiada, pero parece que no hubo complicaciones durante el mismo. Durante el primer año de vida de la niña, los padres no notaron nada que merezca ser destacado. Comenzó a andar a los 15 meses. Al cumplir dos años los padres observaron que Elena no seguía un ritmo de desarrollo normal porque tardaba mucho en empezar a hablar. Cuando tenia tres años sus padres recibieron consejos médicos recomendándole que no iniciaran ningún tratamiento y que la dejaran seguir su proceso madurativo. Un año después ante la ausencia de progresos los padres solicitaron una exploración neuropediátrica, cuyos resultados fueron normales (TAC y EEG). En esta ocasión se le diagnosticó "retraso del lenguaje con signos de encefalopatía de causa no aclarada". Otros informes médicos posteriores resaltaron la presencia de "retraso en el lenguaje y psicomotricidad", asl como problemas de atención.

A los cuatro años empezó a asistir a una guarderla y en el momento de iniciar las consultas estaba escolanizada en un centro 
de educación especial. Elena llevaba dos años recibiendo tratamiento logopédico tradicional en su centro escolar, pero sus padres no hablan observado prácticamente ningún avance en su lenguaje.

Sus padres informaron que en casa se comunicaba con gestos y que emitía sonidos pero no se le entendía lo que decía. Prácticamente carecia de conducta verbal y su repertorio general era muy limitado, salvo en el Area de Autonomía en el cual sus padres se habian ocupado de enseñarle las conductas más básicas.

\section{Evaluacion}

Los datos de la evaluación, se tomaron durante seis sesiones distribuidas a lo largo de dos semanas, en sesiones de 50 minutos. La evaluación se dirigió principalmente a: 1. Evaluar la presencia de conductas prerrequisitas para el aprendizaje (atención, imitación y seguimiento de órdenes). 2. Determinar los problemas conductuales mediante observación durante las sesiones clínicas. 3. Determinar su repertorio verbal mediante la observación y obtener datos sobre el porcentaje de respuestas correctas en las relaciones funcionales: Ecoicas, Tactos, Mandos e Intraverbales. 4. Detectar los déficits conductuales mediante la Guía Portage (Bluma, Shearer, Forhman y Hillary, 1978).

Durante las sesiones de evaluación se observaron y registraron la ocurrencia de dos conductas problema: 1. Respuestas Negativas a seguir las instrucciones del Terapeuta. 2. Levantarse de la silla e interrumpir la tarea.

Por otro lado, para evaluar su repertorio verbal se confeccionó una lista con ejemplos de las cuatro relaciones funcionales citadas anteriormente y se registró el porcentaje de conductas correctas evaluadas. Durante la evaluación se comprobó que únicamente imitaba correctamente los grupos consonánticos: [te], [de] y [be], y las palabras mamá, papá y tata. No obstante, se registró su lenguaje expontáneo y se comprobó que Elena pronunciaba correctamente algunos sonidos: [I], [k], [l] [s], [r], [g], y [n], aunque no los imitaba cuando se le requería que repitiese algún grupo consonántico o palabra que incluyera dichos fonemas. 
Por último, durante las sesiones de evaluación se aplicó te Guía Portage y se comprobó que Elena presentaba timitaciones en las 5 áreas evaluadas y que su nivel de desarrollo era inferior a su edad cronológica. En el Area del Lenguaje su repertorio era equivalente a una niña de 2 años aproximadamente, puesto que no habia conseguido aprender las habilidades verbales propias de esta edad. En el Area Cognitiva se comprobó que únicamente tenía dominadas las conductas correspondientes a una niña de 2 años de edad. En el Area Social mostraba un repertorio equivalente a una niña de 3 años. En el Area Motora únicamente tenía dominadas las destrezas correspondientes a una edad de desarrollo de 3 años, sus déficits se referían a habilidades motoras finas (coordinación oculo-manual).

Por tanto, se puede afirmar que Elena cumplía los criterios propuestos por el DSM-IV para el diágnostico de retraso mental y dado que sus limitaciones lingülsticas impidieron la pasación de la Escala de Inteligencia de Wechsler, puede recibir el diagnóstico de "Retraso Mental de gravedad no especificada".

\section{Tratamiento}

La intervención que se planificó se dirigió a la consecución de los siguientes objetivos:

1. Incrementar los repertorios de atención, imitación motora y seguimiento de órdenes al menos hasta el $80 \%$ de respuestas correctas.

2. Reducir la frecuencia de los problemas de conducta: respuestas negativas a seguir las instrucciones del Terapeuta $\theta$ Interrumpir las tareas levantándose de la silla.

3. Ampliar su repertorio de conducta verbal tanto a nivel ecoico como funcional.

4. Adquisición de conceptos básicos como: tamaños, cantidad, posiciones y colores.

5. Incrementar su repertorio social.

6. Mejorar sus habilidades motoras finas.

El tratamiento fue llevado a cabo por una psicóloga y un observador (estudiante de psicologla) que registraba las respuestas de la 
niña. Para la aplicación del tratamiento se realizaron tres sesiones semanales durante 14 meses en una consulta privada.

Durante el tratamiento también se emplearon algunas ayudas que se describen a continuación y conforme se estabilizaron las respuestas correctas se desvanecieron las ayudas. Para la consecución de cada objetivo, en un primer momento, se aplicó una economía de fichas, que posteriormente eran canjeadas por juegos, actividades o golosinas. Cuando la niña superaba el criterio del $80 \%$ de respuesta correctas se desvanecian las fichas y se aplicaba únicamente reforzamiento social intermitente.

El primer mes de tratamiento se dedicó, entre otras cuestiones, a la consecución de los objetivos 1 y 2 . El procedimiento empleado fue el siguiente:

- Para incrementar la conducta de atención de permanecer sentada se le daba la orden "siéntate" y si en el plazo de 5 segundos no lo hacia, se le guiaba físicamente para que lo hiciera (ayuda demorada). Cuando ella sola se sentaba sin requerir ayuda se le reforzaba diciéndole "muy bien" y dándole una ficha.

Para reducir la frecuencia de la conducta problema de levantarse de la silla $\theta$ interrumpir las tareas cada vez que esto ocurría durante la sesión se actuaba de forma inmediata impidiendo físicamente que se retirara de la silla, se le daba la orden para que se volviese a sentar y se le guiaba para que continuara con la tarea si era necesario. Adicionalmente, de forma paulatina se le reforzó por permanecer sentada tiempos cada vez más largos, de manera que si cumplía el tiempo que se fijaba como objetivo se le pedía que se levantara para jugar (a la pelota, a saltar, con un globo, etc.)

- Para incrementar la frecuencia de mirar al Terapeuta antes de iniciar cualquier tarea o actividad el Terapeuta le decía "mírame" y si trascurridos 5 segundos no lo hacía, el Terapeuta le giraba manualmente la cara para conseguirlo. Cuando ella miraba al Terapeuta sin ayuda se le reforzaba con fichas y elogios. Posteriormente, se reforź la conducta de mirar al Terapeuta de forma espontánea.

- Para conseguir la imitación movimientos de la boca y de la lengua se aplicó un procedimiento de moldeamiento, reforzando aproximaciones sucesivas a la topografía de la respuesta que el 
Terapeuta presentaba como modelo. De esta forma se entrenó a Elena para que sacara la lengua, abriera la boca, cerrara los labios, otc.

- Para incrementar el Seguimiento de órdenes se le daba una instrucción y si respondía negándose con la cabeza, a continuación se le volvía a repetir la instrucción y se le daba guía física para que la cumpliera. Cuando ella segula la orden sin ayuda se le reforzaba.

El tercer objetivo referente a ampliar su repertorio de conducta verbal se inició en la cuarta semana de intervención. Para poder incrementar su lenguaje funcional fue necesario previamente ampliar su repertorio ecoico dadas las carencias que presentaba. Para esto se comenzó reforzando la imitación de las dos palabras que ya imitaba Elena (papá y tata). Posteriormente, se le pidió que imitara la palabra pero con una pausa entre ambas sílabas. Para esto el Terapeuta le requiria: "dl esto pa /pausa / pa". Se reforzó diferencialmente la imitación correcta de las sflabas separadas temporalmente y cuando se consiguió el criterio del $80 \%$ de respuesta correctas, entonces el Terapeuta comenzó a realizar ensayos de imitación de las dos sílabas por separado en dos ensayos diferentes. Es decir, el Terapeuta le pedía "dl esto pa", la niña daba su respuesta y a continuación el Terapeuta le volvla a pedir "dlesto $p a$ " y cuando la niña lo imitaba correctamente se le reforzaba. Este mismo procedimiento se siguló con la palabra "tata" hasta conseguir que imitara por separado ambas śllabas. A continuación, se empleó un procedimiento de moldeamiento para conseguir que Elena imitara sílabas formadas por estos dos sonidos combinados con el resto de las vocales, asi como, los grupos [ma], [no] y [ne] siguiendo el procedimiento empleado en Investigaciones anteriores (Vives y Luciano, 1996).

El hecho de conseguir que Elena fuera imitando un número mayor de sillabas permitio al final del segundo mes de tratamiento que se comenzara a entrenar la imitación de palabras nuevas formadas por grupos consonántlcos que ya imitaba como eran: pato, mano, papel. Por ejemplo el terapueta le pedía: "di esto pato" y posteriormente silabeaba la palabra para que la niña imitara sílaba por sílaba.

En el tercer mes de tratamiento se continuó entrenando la imitación de los grupos: [pa], [pi], [mi], [me], [ta], [ti], [to], [tu], [la], [l i], [no] y [la]. 
Además, se inició el entrenamiento de algunos tactos como: pie, pato, patata, pelo, pollo, bota, tele, llave. Para esto, por ejemplo el Terapeuta, le enseñaba un pato y le preguntaba a la niña: ¿qué es esto?, di "pato". De esta forma se empleó la imitaclón vocal como ayuda para enseñar a la niña a nombrar objetos.

En el cuarto mes de tratamiento se continuó entrenando la imitación de los grupos consonánticos con sonidos que Elena ya pronunclaba en su lenguaje espontáneo, pero que no imitaba, como eran eran [l], [k], [l] [s], [r], [g], [n] y [b]. Para esto se reforzó diferencialmente la imitación correcta de sílabas con estos sonidos. También se introdujo el entrenamiento de algunos tactos de palabras de uso común: codo, huevo, boca, limón, bola, pera, pelota, galleta, tomate, silla, agua, bici, cama, goma, moto.

En el quinto mes se comenzó el entrenamiento en algunos mandos en los que se requería que emplease las palabras que ya sabía imitar. Para esto se le enseñaba por ejemplo una patata frita y se le decía: "pidemela, di esto: dame patata" y la niña lo imitaba. Paulatinamente se fue desvaneciendo esta ayuda hasta conseguir que lo pidiera cuando se le mostraban patatas.

En el sexto mes se inició el moldeamiento de sonidos que no pronunciaba como: [x], [c], [f], y se hicieron ensayos para mantener la imitación de los sonidos ya enseñados. Se continuó con el entrenamiento de mandos y tactos con palabras funcionales de uso común.

En el séptimo mes se prosiguió el entrenamiento de los sonidos $[x],[c],[f]$, as[ como [? ] y se enseñaron palabras nuevas con estos sonidos: leche, jamón, coche, zapato, foto, ojo. Estas palabras se entrenaron también en otras relaciones funclonales como mandos y tactos. También se entrenaron algunos conceptos como grande y pequeño mediante un procedimiento de discriminación sin error, siguiendo las pautas de Lovaas (1977).

En el octavo y noveno mes se ampliaron los mandos entrenados y se crearon las condiciones apropiadas para enseñar a la niña que hiciera peticiones con las consignas: "quiero..(objeto)"; "vamos a ... (acción)"; y "ayúdame". Se le enseñó a nombrar acciones comunes mostradas en fotos como: beber, comer, bailar, pintar, saltar, salir. 
Para esto se le mostraba una foto y se le preguntaba " $¿ q u e ́$ hace este niño?", dándole a continuación la respuesta que tenla que imitar.

En el décimo mes se amplió el entrenamiento de tactos de objetos comunes (caballo, vaso, ficha, queso, toalla, pantalón, dientes, ducha, etc.) y de acciones como: cortar, ver la tele, jugar, comprar, cantar, bañar, lavar. Además se aprovechaban las situaciones en las que Elena estaba haciendo alguna actividad para preguntarle "¿qué haces?" y se le daba la respuesta para que la imitara. Posteriormente se desvanecia la ayuda ecoica.

En el undécimo mes amplió el entrenamiento de mandos, tactos de objetos y acciones diferentes. Se comenzó a enseñar a la niña a responder a las preguntas "¿qué tienes?" y "¿qué tiene?". Para esto se empleó al principio ayuda ecoica que posteriormente fue desvanecida. Se enseñó la discriminación de conceptos: poco/mucho.

Desde el duodécimo mes hasta el décimo cuarto se realizó el entrenamiento de respuestas intraverbales. Para esto, de forma paulatina se le plateaba a Elena ciertas preguntas comenzando con las consignas: ¿de quien?; ¿con quien?; ¿para quién?; ¿dónde está?; ¿cuántos?; ¿para que?; ¿cómo?. Para llevar a cabo parte del tratamieno el Terapeuta le decía a Elena por ejemplo: "¿de quien es esto?, di mío". Paulatinamente se fue retirando la ayuda ecoica y se continuaron los ensayos de entrenamiento con cada una de las preguntas hasta conseguir el $80 \%$ de respuestas.

También desde el duodécimo mes se llevó a cabo el entrenamiento en discriminación de conceptos: encima/debajo; dentro/fuera; algunos colores (rojo y amarillo). También se le enseñó a contar 3 objetos empleando ayudas ecoicas y posteriormente hasta 5 objetos.

Los objetivos referentes al Area Soclal se entrenaron en colaboración con los padres, a los que se les proporcionaba pautas sobre cómo entrenar los objetivos del área social de la Gula Portage: espera su turno; emplear "por favor" y "graclas"; saludar y despedirse; pedir permiso en situaciones adecuadas; y obedecer a los padres. Una vez en semana se revisaban con ellos los progresos que iba registrando Elena y se resolvían las dificultades que iban encontrando los padres, para lo cual se realizaban 
ensayos con Elena mostrándoles las forma de hacerlo y posteriormente los padres ponian en práctica las indicaciones del Terapeuta.

El último objetivo de tratamiento se comenzó a trabajarlo a partir del sexto mes para lo cual se dedicaron una parte de las sesiones a mejorar las habilidades motoras finas de Elena. Las habilidades entrenadas fueron: cortar con tijeras; botar la pelota; coger el lápiz correctamente; colorear; dibujar; atornillar objetos; y saltar sobre un pie. Para este entrenamiento se aplicaron procedimientos de moldeamiento.

\section{RESULTADOS}

Los resultados obtenidos durante las sesiones de evaluación respecto a las conductas prerrequisitas están detallados en la Tabla 1 y fueron los siguientes:

- Conductas de atención: la media se situó en el $45 \%$ de respuestas correctas de las respuestas de atención, en las que se incluía: sentarse ante la orden correspondiente, solo lo realizó el $40 \%$ de las ocasiones en las que se requirió; y contacto ocular con el Terapeuta, solo ocurrió el $50 \%$ de las ocasiones en las que se requirio.

- Imitación de movimientos. solo se produjeron el $60 \%$ de las veces en las que se le solicitó que imitara algún movimiento corporal. Se observó que no solía imitar movimientos de la boca ni de la lengua, aunque si lo hacía cuando se trataba de movimientos de la manos o pies.

- Seguimiento de órdenes: solo realizó el $70 \%$ de las órdenes que se le requirieron, y al resto respondía con movimientos de negación con la cabeza.

En las sesiones de evaluación se observaron dos conductas problema: 1\%. Respuestas negativas a seguir las instrucciones del Terapeuta con una frecuencia que oscilaba entre 7 y 10 veces por sesión. 2a. Levantarse de la silla e interrumpir la tarea que ocurría con una frecuencia de 7 a 12 veces por sesión. 
Tabla 1.- Resultados de la Evaluación Iniclal

\begin{tabular}{|c|c|c|}
\hline Area & Componente & $\begin{array}{c}\text { Porcentaje } \\
\text { de Resp. Correctas } \\
\end{array}$ \\
\hline $\begin{array}{l}\text { Conductas } \\
\text { prerrequisitas }\end{array}$ & $\begin{array}{l}\text { Conductas de atención } \\
\text { Imitación de movimientos } \\
\text { Seguimiento de órdenes }\end{array}$ & $\begin{array}{l}45 \\
60 \\
70 \\
\end{array}$ \\
\hline $\begin{array}{l}\text { Conducta Verbal } \\
\text { Relaciones funcionales }\end{array}$ & $\begin{array}{l}\text { Ecoicas } \\
\text { Tactos } \\
\text { Mandos } \\
\text { Intraverbales } \\
\end{array}$ & $\begin{array}{l}10 \\
10 \\
30 \\
15 \\
\end{array}$ \\
\hline Guia Portage & $\begin{array}{l}\text { Lenguaje } \\
\text { Cognitiva } \\
\text { Social } \\
\text { Motore } \\
\text { Autonomia }\end{array}$ & $\begin{array}{l}15 \\
20 \\
45 \\
60 \\
70\end{array}$ \\
\hline
\end{tabular}

En la Tabla 1 se puede comprobar que en la primera evaluación Elena sólo imitaba correctamente el $10 \%$ de los grupos consonánticos requeridos (Respuestas Ecoicas).

El tratamiento se aplicó durante 140 sesiones de 50 minutos de duración. Para esto, la niña asistía a consulta 3 veces en semana.

Los resultados del tratamiento de las conductas prerrequisitas se detallan en ia Figura 1. En la parte superior se puede observar que las conductas prerrequisitas de atención se incrementaron y llegaron a una tasa superior al $\mathbf{8 0 \%}$ de respuestas correctas. Para las conductas de sentarse ante la orden del Terapeuta y de imitar posiciones de la boca se consiguió el criterio de aprendizaje en pocas sesiones, mientras que la conducta de mirar al Terapeuta no se estabilizó hasta la sesión 15. El entrenamiento para el seguimiento de órdenes se consiguió en dos sesiones porque se partía del $70 \%$ de respuestas correctas, pero estos datos no se han graficado para facilitar la visión de los resultados. No obstante, ésta intervención 
estaba relacionada con la aplicada para la reducción de conductas negativas que se muestra en la parte inferior de la Figura 1. En esta gráfica los cinco primeros datos corresponden a la Línea Base y posteriormente se observa los efectos del tratamiento. A partir la sesión 23, prácticamente se eliminan las conductas negativas a seguir las órdenes del Terapeuta y la conducta de levantarse de la silla durante los ensayos de entrenamiento.
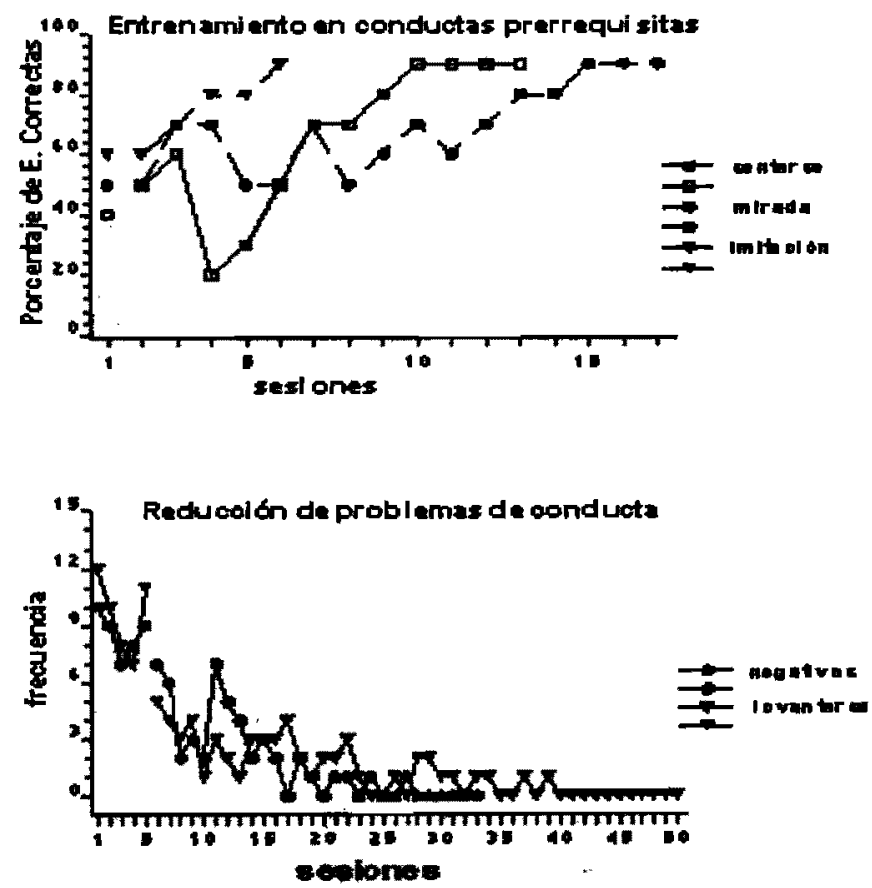

Figura 1.- Superlor: Porcentaje de Respuestas Correctas durante el entrenamiento en conductas prerrequlsitas; Inferlor: Frocuencla de problemas de conducta por sesión durante las sesiones do tratamlento 
En la parte superior de la Figura 2 puede observarse los datos Post-test de las cinco relaciones funcionales de conducta verbal. Se observa un incremento superior en el porcentaje de Respuestas Ecoicas, Tactos y Mandos, dado que el tratamiento se focalizó principalmente en estas tres relaciones funcionales. En la parte inferior de la Figura 2 se comprueba que se incrementaron los resultados de la Guia Portage en la segunda pasación realizada después del tratamiento. El Area del Lenguaje fue la que registró un incremento mayor en el porcentaje de respuestas correctas, puesto que los objetivos de tratamiento se centraron principalmente en esta área.
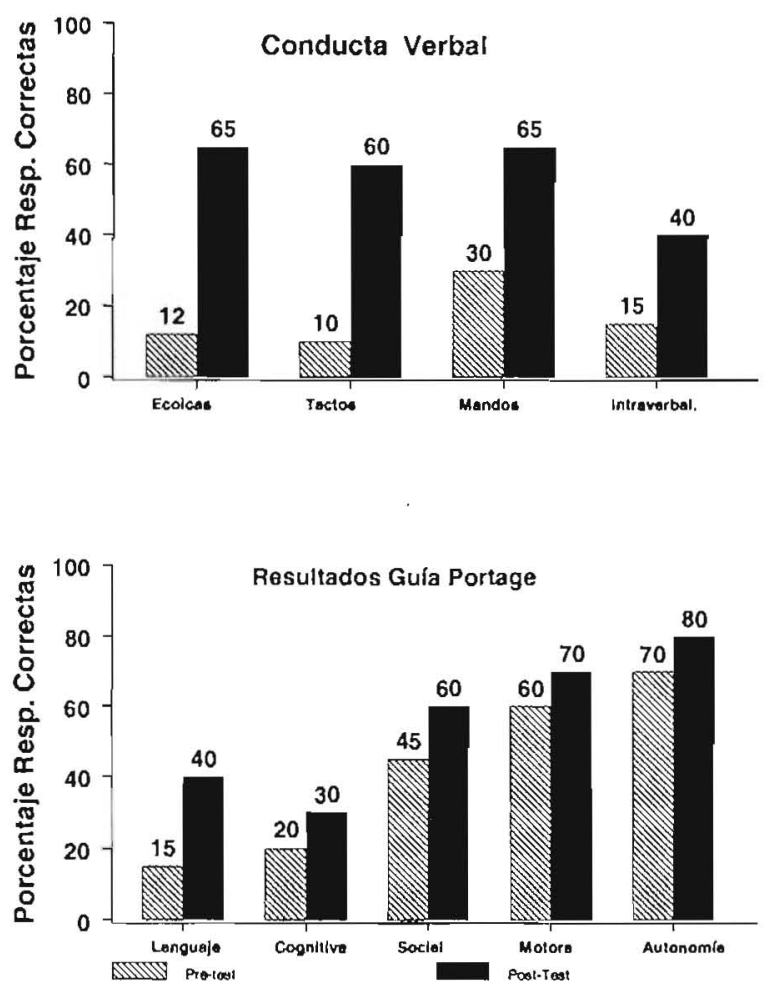

Figura 2.- Comparación de resultados Pre-test y Post-test del repertorio verbal (parte superior) y de la pasación de la Guía Portage (inferior) 


\section{CONCLUSIONES}

En este estudio se comprueba que al aplicar un tratamiento conductual se consigue enseñar a hablar a una niña con retraso generalizado que anteriormente habla recibido otro tipo de tratamiento sin obtener resultados. Elena carecla prácticamente de un repertorio imitativo vocal y de lenguaje funcional a pesar de haber recibido durante dos años un tratamiento logopédico estandarizado. Los resultados de la evaluación realizada para este estudio fueron el fundamento para planificar la intervención que se centró en los déficits y excesos conductuales detectados. La intervención se inició con la adquisición de conductas prerrequisitas, la reducción de sus conductas problema y el entrenamiento de repertorios de Imitación Vocal. Esta fase de tratamiento previa facilitó la adquisición del lenguaje funcional básico que consiguió después de 14 meses de tratamiento conductual. Por tanto, se comprobó que la inclusión de las conductas prerrequisitas entre los objetivos de tratamiento y la aplicación de los procedimientos de intervención conductuales (Guess, Sailor y Baer, 1974, 1976; Kent, Basil y del Rio 1982; Lovaas, 1977; Maurice, Green y Luce , 1996; Vives y Luciano 1996, entre otros,) hicieron posible el aprendizaje de otras conductas más complejas que esta niña no había alcanzado mediante un tratamiento tradicional más prolongado que el empleado en este estudio.

Los resultados de la evaluación pusieron de manifiesto que Elena presentaba Retraso en su desarrollo que era más grave en el Area del Lenguaje y que presentaba déficits en las conductas prerrequisitas para el aprendizaje, así como la presencia de problemas de conducta. Ante estos resultados se consideró necesario comenzar por ampliar su repertorio de conductas prerrequisitas y reducir las conductas problema. Posteriormente, se llevo a cabo el tratamiento en el Area del Lenguaje, y entonces, el primer objetivo fue incrementar su repertorio imitativo para que fuese posible posteriormente aumentar su lenguaje funcional. Para esto se comenzó reforzando la imitación de las dos palabras que fue capaz de repetir correctamente durante la evaluación y mediante un procedimiento de moldeamiento se consiguió la imitación de silabas separadas y posteriormente se consiguió la imitación de otras sflabas. La adquisición de la imitación 
vocal permitió que la niña pudiese aprender palabras nuevas y posteriormente en otras relaciones funcionales como Tactos y Mandos. En la última parte del tratamiento se introdujo el entrenamiento de Intraverbales, algunos conceptos básicos y conductas motoras. EI tratamiento se coordinó con los padres para entrenar los objetivos referentes al Area Social y de Autonomía. En la Evaluación Posttratamiento se comprobó un incremento en su lenguaje productivo y funcional que se reflejó también en los resultados de la aplicación de la Gula Portage, en la cual se detectó un incremento en el porcentaje de conductas verbales que tenla adquiridas y en las Areas Cognitiva, Social y Motora principalmente.

Elena continuó recibiendo tratamiento en el mismo centro durante dos años más, pero los datos posteriores no han sido incluidos porque están incompletos debido a que el tratamiento se amplib́, se diversificó por el cambio de Terapeuta y se complicó la toma de datos por las ausencias de observadores. De todas formas, las evaluaciones posteriores reflejan que Elena amplió su vocabulario y su lenguaje funcional (Mandos e Intraverbales) y consiguió seguir conversaciones básicas. Cuando los padres interrumpieron el tratamiento Elena presentaba un repertorio lingũistico básico que le permitla cumplir las funciones comunicativas principales y unos niveles de autonomía personal aceptables.

En este estudio se pone de manifiesto que Elena fue capaz de aprender cuando se aplicaron los procedimientos del Análisis Conductual Aplicado, a pesar de haber fracasado otro tratamiento tradicional. Este estudio confirma las afirmaciones realizadas por Wicks-Nelson e Israel (1997) referentes a que la aproximación conductual han tenido un gran éxito en la enseñanza de niños con retraso a los que se les había considerado incapaces de aprender.

A pesar de todo, este estudio hubiese quedado más completo si se hubiese podido aportar más información del tratamiento que habla recibido esta niña con anterioridad y asI poder contrastar los datos, pero lamentablemente esto no fue posible. No obstante, se echan en falta estudios de este tipo en nuestro Idloma, en los que se aporten conocimientos prácticos a los profesionales que desconocen estos procedimientos del Anállsis Conductual Aplicado que han demostrado ser útiles y efectivos en la intervención con personas con retraso. 


\section{BIBUOGRAFIA}

American Assoclation of Mental Retardation, (2002/2004). Retraso Mental. Definición, clasificación y sistemas de apoyo. Madrid: Allanza Editorial.

American Psychatric Ascoclation (2002). DSM-IV-TR. Manual de Diagnóstico y Estadístico de los Trastomos Mentales. Barcelona: Masson.

Baer, D.M., Peterson R.F. y Sherman, J. A. (1967). The development of imitation in young children. Joumal of Experimental Child Psychology, 1, 37-49.

Bljou, S.W. (1976/1982). Pslcología del Desamollo infantil. Volumen 3. México: Trillas.

Bluma, S.M.; Shearer, M.S.; Frohman, A.H. y Hlllard, J.M. (1978). Gula Portage de Educación Preescolar. Madrid: TEA Ediciones, S.A.

Drasgow, E., Halle, J.W. y Ostrosky M.M. (1998) Effects of differential reinforcement on the generalization of replacement mand in three children with severe language delays. Joumal of Applied Behavior Analysis, 31, 357- 374.

Del Rlo, M.J., Vllaseca, R. y Gràcla, M. (1997). La interacción y el desarrollo comunicativo y lingüístico en niños con deficiencla mental. En $M$. J. del Río (Ed.). Lenguajo y comunicación en personas con nocesidades especiales (pp.113-160). Barcelona: Martínez-Roca.

Gallndo, E., Bernal, T., HIno|osa, G., Galguera, M. I., Taracena, E., Padllla, F. (1980). Modificación de conducta en educación especial. México: Trillas.

Garcla, E., Baer, D.M. y Firestone, 1. (1971). The development of generalized imitation within topographically determined boundaries. Joumal of Applied Behavior Analysis, 4, 101-112.

Gll Roalee-Nieto, J. (1988) Evaluación y diagnóstico conductual en el retraso en el desarrollo En M.C. Luclano y J. GH (Eds.) Análisis e intervención conductual en Retraso on el desarrollo (pp. 25-50). Granada: Servicio de Publicaciones de la Universidad de Granada.

Green, G. (1996) Evaluating claims about treatments for autism. En C. Maurice, G.Green y S.C. Luce (Eds). Behavbr interventions for young children with autism (pp.15-28) Austin: Pro Ed.

Guess, D., Sallor, W. y Beer, D.M. (1974). To teach language to retarded children. En R.L. Schiefelbusch y L.L. Lloyd (Eds). Language perspectives:Adquisition, Retardation and Intervention (pp. 61-83). Baltimore: University Park Press.

Guess, D., Sallor, W. y Baer, D.M. (1976). A functional speech and language program for the severely retarded. Lawrence: H\&h Enterprises.

Guess, D., Keogh, W. y Sallor, W. (1986). Generalización del habla y de 
la conducta lingüistica. En R. L. Schiefelbusch (Ed). Bases de la Intervención en el Lenguaje (pp. 303-320). Madrid: Alhambra Universidad. Harris, S.L. (1976). Managing Behavior. Behavior Modification: Teaching Speech to a Nonvertal Child. Lawrence, Kansas: H. and H. Enterprises. Kent, R.L., Basll, C. y del Rlo, M. J. (1982). Programa para la adquisición de las primeras etapas del lenguaje. Madrid: Siglo XXI.

Lovaas, O.l. (1977/ 1981). El niño autista. Madrid: Ediciones Morata.

Lovaas, O.I. (1990). Enseñanza de niños con trastomos del desarrollo. Barcelona: Martínez Roca.

Luclano, M. C. (1984). Implantación de conducta verbal en niños no verbales. Revista Española de Pedagogla, 164-165, 257-282.

Luclano, M. C. (1997). Intervención psicológica en retraso en el desarrollo: una perspectiva funcional. En M. C. Luciano (Ed.). Manual de Psicologia Clínica (pp. 467-525). Valencia: Promolibro.

Luclano, M. C. y Polalno-Lorente, A. (1986) Effects of adquisition of prerrequisite behavior on the leaming of nonvocal verbal behavior and vocal imitation in children with severe retardation. The Psychological Record, 36, 315-332.

Luclano, M.C.; Lopez, F. y Vives, M.C. (1997). Retraso en el desarrollo. Definición y aproximaciones explicativas. En M.C. Luciano (Ed.). Manual de Psicologla Clinica. Infancia y adolescencia. (pp. 433- 463). Valencia: Promolibro.

Maurlce C, Green G. y Luce S.C. (1996). Behavior interventions for young children with autism. Austin, TX: Pro Ed.

McCoy, J. F. y Buckhalt, J. A. (1981). Languaje adquisition. En J.L. Matson y J. R. McCartney (Eds.). Handbook of behavior modification with the mentally retarded. New York: Plenum Press.

Ribes, E. (1972). Técnicas de modificación de conducta. Su aplicación al retardo en el desarrollo. México: Trillas.

SkInner, B.F. (1957/1981). Conducta verbal. México: Trillas.

Smlth, T. (1996). Are other treatment effective?. En C. Maurice, G.Green y S.C. Luce (Eds). Behavior interventions for young children with autism (pp.: 45-59) Austin, TX: Pro Ed.

Sloane, H.N, Johnston, M.K. y Harris, F.R. (1968). Remedial procedure for teaching verbel behavior to speech deficient or defective young children. En H.N. Sloane, y B.D. MacAulay (Eds.). Operant procedures in remedial speech and language training (pp.: 77-101). Boston: Houghton and Mifflin Company.

Sundberg, M. L. Endicott, K.y Elgenheer, P. (2000). Using intraverbal prompts to establish tacts for children with autism. The Analysis of Verbal Behavior, 17, 89-104. 
Sundberg, M. L y Michael, J. (2001). The benefits of Skinner's Analysis of Verbal Behavior for Children with autism. Behavior Modification, 25, 698-724..

Vives, M. C. y Luclano, M.C. (1996). Mejorando la implementación de un procedimiento para la corrección de topografías vocales. Psicothema, 8, 563-572.

Vives, M. C., y Luciano, M. C. y Valero, L. (2001): Efectos de Generalización en el tratamiento de trastomos de articulación vocal. Psicothema, 13, 101-110.

Vives, M. C., y Luciano, M. C. y Valero, L. (2002): Influencia de dos procedimientos sobre la generalización de la corrección de errores articulatorios Psicothema, 14, 144-153

WatkIns, C.L. Pack-Telxteira, L. y Howard J.S. (1989). Teaching intraverbal behavior to severely retarded children. The Analysis of Vertal Behavior, 7, 69-81.

Wicks-Nelson, R. Israel, A.C. (1997): Retraso Mental. En R. WicksNelson y A.C. Israel Psicopatogía del niño y del adolescente. (pp.: 237269). Madrid:Prentice-Hall 